\title{
Dye-doped Silica Nano-powder Random laser Emission
}

\author{
Li Wenchao \\ School of Control and Engineering, \\ Northeastern University at \\ Qinhuangdao, \\ Qinhuangdao, 066004, P. R. China
}

\author{
Sha Xiaopeng \\ Institute of Electrical Engineering, \\ Yanshan University, Qinhuangdao, \\ 066004, P. R. China
}

\author{
Sun Yuchao \\ Institute of Electrical Engineering, \\ Yanshan University, Qinhuangdao, \\ 066004, P. R. China
}

\section{Li Zhiquan}

Institute of Electrical Engineering, Yanshan University, Qinhuangdao, 066004, P. R. China

\author{
Li Xin \\ Control and Simulating Center, School \\ of Astronautics, Harbin Institute of \\ Technology, \\ Harbin, 150080, P. R. China
}

\section{Du Yiying}

Institute of Electrical Engineering, Yanshan University, Qinhuangdao, 066004, P. R. China

\begin{abstract}
We have researched the relationship between the luminescence properties, concentration changes, particle size of fluorescent particles and the additional scattering particles, and the random lasing threshold of a new disorder media. Maxwell equations and the rate equations are solved by using the finite difference time domain (FDTD) method directly. We calculate the emission line and the dependence of lasing threshold on the concentration. When Rh6G-SiO2 occupies $4 \mathrm{wt} \%$ of the sample, the threshold is $8.5 \mu \mathrm{J} /$ pulse; the impact of the added small scatterers $\mathrm{TiO}_{2}$ on random laser emission is such little that it could be negligible. In addition, the lasing threshold properties of random media under different condition are calculated, and the impact of these factors on the random laser emission is proposed. Finally, the variation of the concentration of fluorescence particles has little influence on random lasing threshold which is immune to external addition of scatterers.
\end{abstract}

Key words-Random laser; FDTD ; multiple-scattering ; laser-dyes

\section{INTRODUCTION}

Since Letkhov first proposed that there is laser emission behavior in random gain medium in 1967, random lasers have attracted attention of many scholars with its unique light-emitting characteristics and physical mechanisms. Random laser is a system formed by elastic scatterers with random distribution, embedded in the optical gain medium [2].The multiple scattering of light in the optical media replaces the traditional laser optical cavity. At present, in the random laser experiments, a number of random laser emission phenomena of organic-inorganic disordered structure media are observed (such as the spread nanoparticles laser dye, neodymium that doped with crystalline powder, ceramic, polymer, semiconductor nanoparticles, organic biological tissue, and liquid crystal) [3-5]. Each specific features and form of random media lead to the special feedback mechanism, so it is difficult to use the same theory to describe all random laser phenomena.
In addition, compared with conventional lasers, random lasers have small size (up to now the reported smallest minimum size is $1.5 \mu \mathrm{m}[6]$ ), easy to fabricate (eliminating the optical collimation), low cost and convenient mode of regulation. These advantages in the new optical systems and devices will play a further important role, and make innovative achievements $[7,8]$.

This paper studied the characteristics of dye random lasers The gain medium is dye solution of organic fluorescent particles and water or organic solvents. The dye has a large emission, absorption cross-section (10-20-10-19m2) and high fluorescence quantum efficiency $(0.7-0.8)$. Therefore a very high gain can be effectively pumped. In addition, the selecting of dye as the gain medium has another advantage: the ability to form a so-called four-level system. Two-level system will limit the reversal rate in $50 \%$ due to saturation, so it can not produce reversal particles what laser emission needs through resonant optical absorption. The media is pumped optically, as the multiple scattering effect, and the light will be confined in the internal medium for a long time. Then the strong amplification opportunity which has the significant role of gain is achieved. A recent study reported a new disordered structure of a random laser emission $[9,10]$. In this paper, we use FDTD method to analyze the influence to the random laser pulse by doped inert particles in the above structure of the random medium and the variation of concentration.

\section{GAIN MEDIUM AND THEORETICAL MODEL}

In Figure 1, the random medium structure is set: uniform square with length $L$ and refractive index $n_{1}$, the radius of the medium particle is $r$, the refractive index is $\mathrm{n}$, the gain medium corresponds to the dye random lasers. 


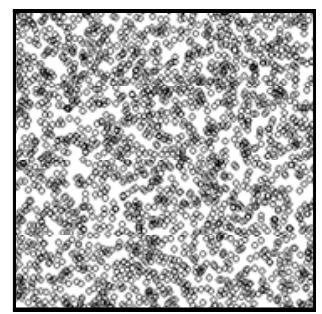

Fig. 1. Spatial distribution of scattering nanoarticles in random medium on $\mathrm{x}-\mathrm{y}$ plane.

Then we establish a simplified model to study the characteristics of quasi-state mode. The FDTD method is employed to solve the Maxwell equations directly around the PML (Perfect Matched Layer) boundary conditions and calculate the field strength at various points in the media of different time. Then the spatial distribution and time evolution characteristics of the quasi-state modes in the medium are obtained. We will be able to get the spectrum characteristics of quasi-state modes by Fourier transform.

Two-dimensional transverse magnetic (TM) mode is studied and TM-wave electric field and magnetic field components satisfy the following Maxwell equations:

$$
\begin{gathered}
\frac{1}{\mu_{0}} \frac{\partial E_{z}}{\partial y}=-\frac{\partial H_{x}}{\partial t} \\
\frac{1}{\mu_{0}} \frac{\partial E_{z}}{\partial x}=\frac{\partial H_{y}}{\partial t} \\
\varepsilon_{i} \varepsilon_{0} \frac{\partial E_{z}}{\partial t}+\frac{\partial P_{z}}{\partial t}=\frac{\partial H_{y}}{\partial x}-\frac{\partial H_{x}}{\partial y}
\end{gathered}
$$

Where $\varepsilon_{0}$ and $\mu_{0}$ are the vacuum permittivity and vacuum permeability, $\varepsilon_{i}=n_{i}^{2}, i=1,2$, and $P_{z}$ is the polarization of $\mathrm{z}$ direction.

The four energy level in the laser system are ground state level $\mathrm{E}_{1}$ (pumping process of the low-energy level), pumped higher energy level $E_{4}$, the upper level $E_{3}$ and lower level $E_{2}$. The particle in the ground state energy level is transited from $\mathrm{E}_{1}$ to $\mathrm{E}_{4}$ with the pumping rate is $W_{p}$; Particles reached to $\mathrm{E}_{4}$ very quickly transferred to the upper level $E_{3}$ in the form of non-radiative transition of which the probability is $1 / \tau_{43}$.Before the formation of the inversion, the particles in $E_{3}$ return to $E_{2}$ mainly in the form of spontaneous emission and the probability is $1 / \tau_{32}$; the particles in $E_{2}$ return to $E_{1}$ in the form of spontaneous emission with probability $1 / \tau_{21}$.

The particle number density of every energy level is assumed as $N_{i}(i=1,2,3,4)$, therefore, the rate equations of the four-level system are $[11,12]$ :

$$
\begin{aligned}
& \frac{d N_{1}}{d t}=\frac{N_{2}}{\tau_{21}}-W_{p} N_{1} \\
& \frac{d N_{2}}{d t}=\frac{N_{3}}{\tau_{32}}-\frac{N_{2}}{\tau_{21}}-\frac{E_{z}}{\eta \omega_{1}} \frac{d P}{d t}
\end{aligned}
$$

$$
\begin{aligned}
\frac{d N_{3}}{d t} & =\frac{N_{4}}{\tau_{43}}-\frac{N_{3}}{\tau_{32}}-\frac{E_{z}}{\eta \omega_{1}} \frac{d P}{d t} \\
\frac{d N_{4}}{d t} & =-\frac{N_{4}}{\tau_{43}}+W_{p} N_{1}
\end{aligned}
$$

Where $W_{p}$ is pumping rate, $\tau_{21}, \tau_{32}$ and $\tau_{43}$ a re respectively the lifetime of energy level 2, 3 a nd $4, \omega_{1}=\left(E_{3}-E_{2}\right) / \eta$ is the central frequency $o$ $f$ the radiation light and $\left(E_{z} / \eta \omega_{1}\right) d P / d t$ is the rate of stimulated emission.

According to the polarization intensity $\mathrm{P}$, we have

$$
\frac{d^{2} P}{d t^{2}}+\Delta \omega_{1} \frac{d P}{d t}+\omega_{1}^{2} P=\kappa \Delta N E_{z}
$$

$\Delta N=N_{2}-N_{3}$ is the difference of the number of particles between the upper and the lower level in the unit volume. $\Delta N<0$ correspond that particle population inversion occurs as the action of external pumping. $\Delta \omega_{1}=1 / \tau_{32}+2 / T_{2}$ is the full width at half maximum(FWHM) of the laser radiation spectrum. $\mathrm{T}_{2}$ is the atomic relaxation time which is a constant $\kappa=6 \pi \varepsilon_{0} c^{3} /\left(\omega_{1}^{2} \tau_{32}\right)$.

FDTD method to solve the equation (1)-(8), the boundary condition selects PML (perfectly matched layer). The space step length is $\Delta x=\Delta y=10 \mathrm{~nm}$, and the time step is selected $\Delta t=\Delta x /(2 c) \approx 1.67 \times 10^{-17} \mathrm{~s}$ according to the Courant stability conditions.

In the calculation, we employ the statistics of literature $[10,13]$ :

$$
\begin{aligned}
& \tau_{21}=5 \times 10^{-12} \mathrm{~s}, \tau_{32}=10^{-10} \mathrm{~s}, \tau_{43}=10^{-13} \mathrm{~s}, \\
& T_{2}=2.18 \times 10^{-14} \mathrm{~s}, \\
& v_{1}=\frac{\omega_{1}}{2 \pi}=5.64 \times 10^{14} \mathrm{~Hz}\left(\lambda_{0}=532 \mathrm{~nm}\right) 。
\end{aligned}
$$

\section{SIMULATION RESULTS}

At first, we studied the spectrum lines of silica gel containing 4wt \% Rh6G-SiO 2 GP samples in different pump energy. As shown in figure 2, when pump energy was 8 $\mu \mathrm{J} /$ pulse, the emission spectrum appears the typical wide band fluorescence emission. With the increasing of pump energy, the effective emission line width significantly reduced so as to prove the existence of the laser emission. A remarkable characteristic is that the wider tail end caused by photoluminescence was inhibited completely in $100 \mu \mathrm{J} /$ pulse, leaving only narrow emission peak due to the gain (orange curve in figure 2 (a)). Figure 2 (b) indicates that with the increase of the pump pulse energy, line width slowed sharply at some points which named laser pulse threshold of 8.5 $\mathrm{uJ} /$ pulse. 


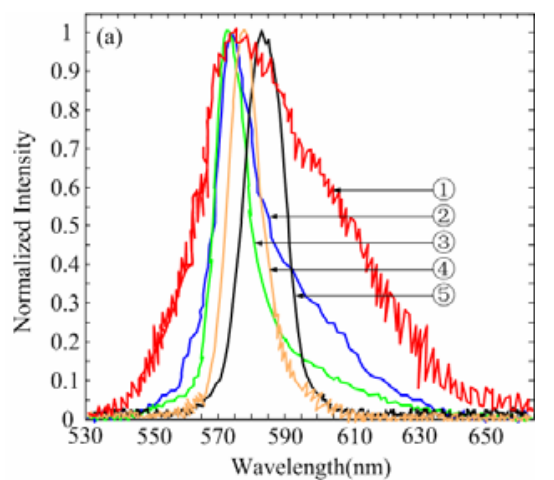

(a)

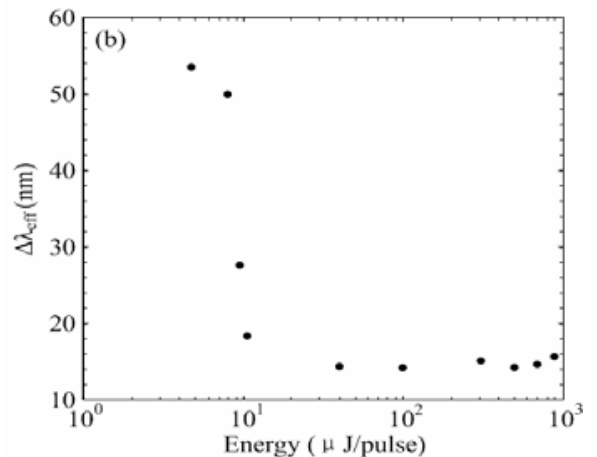

(b)

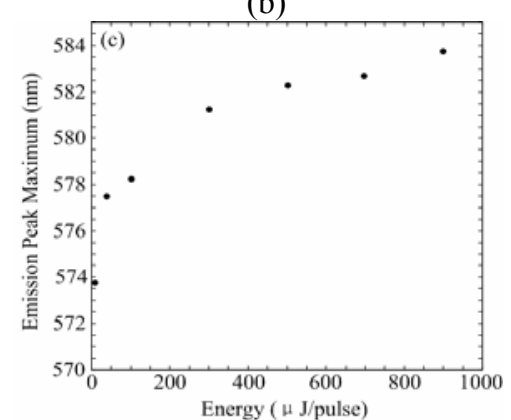

(c)

Fig. 2. Emission spectra with different pump intensity;(a)Normalized emission spectra of the GPs samples of silica gel containing $4 \mathrm{wr} \%$ Rh6G-SiO ${ }_{2}$ nanoparticles obtained at $8 \mu \mathrm{J} /$ pulse (red), $9.5 \mu \mathrm{J} /$ pulse (blue), $11 \mu \mathrm{J} /$ pulse (green), $100 \mu \mathrm{J} /$ pulse (orange) and $900 \mu \mathrm{J} /$ pulse (black). (b)Curve of emission line widths and pump energy. (c)Profile of emission peak and pump energy.

On this basis, we study the effect of the GP samples $\mathrm{Rh} 6 \mathrm{G}-\mathrm{SiO}_{2}$ concentration variation on random laser emission. In figure 3(a) we could see that GP samples of different concentrations of $\mathrm{Rh} 6 \mathrm{G}-\mathrm{SiO}_{2}$ has similar curve, and the only difference was that $0.1 \mathrm{wt} \%$ GP sample threshold is larger. We launch the values of emission efficiency with different density (Fig. 2(b)). It could also be found that laser emission efficiency with GP samples containing 4 wt \% $\mathrm{Rh} 6 \mathrm{G}-\mathrm{SiO}_{2}$ is the highest. In addition, we still could see that in low excitation energy and different GP samples Rh6G-SiO concentration has different output pulse FWHM. It is noteworthy that when $\mathrm{Rh} 6 \mathrm{G}-\mathrm{SiO}_{2}$ concentration is from $0.1 \mathrm{wt}$ $\%(\tau=4.6 n s)$ increased to $2 \mathrm{wt} \%(\tau=6.2 n s)$, the lifetime is also increased; when concentration increased to $5 \mathrm{wt} \%(\tau=5 \mathrm{~ns})$, the lifetime is suddenly reduced. The phenomenon may be due to the existence of re-absorption, non-radiation caused by the formation of dye aggregates and the not activated channel. In the first case, the increasing of the lifetime is due to the radiation trap. The increasing of $\mathrm{Rh} 6 \mathrm{G}-\mathrm{SiO}_{2}$ nanoparticles concentration makes the effect caused by re-absorbing phenomena larger, which explained why the beginning of life tended to increase.

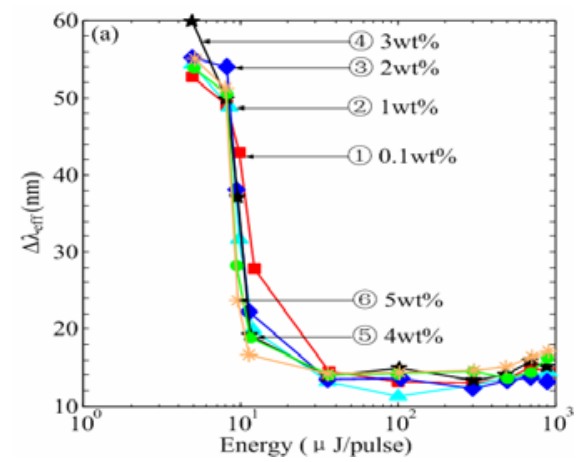

(a)

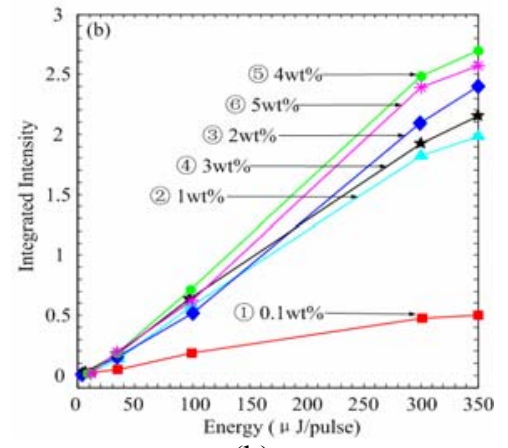

(b)

Fig. 3. (a) In the GP samples of different silica gels containing $0.1 \mathrm{wt} \%$ (red squares), $1 \mathrm{wt} \%$ (turquoise triangles), $2 \mathrm{wt} \%$ (blue diamonds), $3 \mathrm{wt} \%$ (black pentagons), $4 \mathrm{wt} \%$ (green dots), and $5 \mathrm{wt} \%$ (orange snowflakes) $\mathrm{Rh} 6 \mathrm{G}-\mathrm{SiO}_{2}$ nanoparticles, the dependence of effective emission line width on pump energy. (b)Relationship between integrated intensity of the emission spectra and pump pulse energy.

On the contrary, the emergency of the dye combination may be the right reason why the life time suddenly reduced as Rh6G-SiO 2 nanoparticles concentration was for $5 \mathrm{wt} \%$. With the increase of the fluorescent nanoparticles number, the formation of dye aggregation is a slow process, so when the concentration increased to a certain extent, their impact plays a leading role gradually.

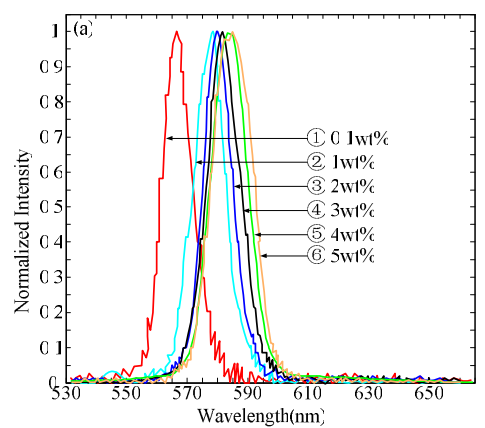

(a) 


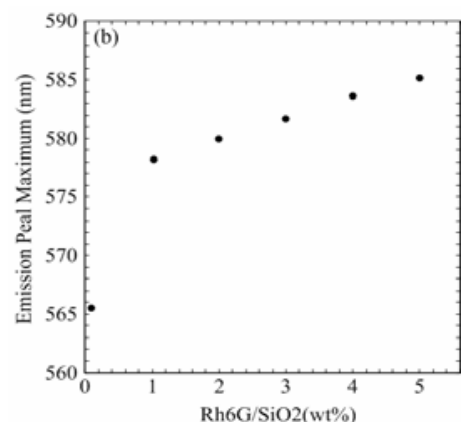

(b)

Fig. 4. (a)Normalized emission spectra of the GP samples of different silica gels containing $0.1 \mathrm{wt} \%$ (red), $1 \mathrm{wt} \%$ (turquoise), $2 \mathrm{wt} \%$ (blue), $3 \mathrm{wt} \%$ (black) $4 \mathrm{wt} \%$ (green), and $5 \mathrm{wt} \%$ (orange) $\mathrm{Rh} 6 \mathrm{G}-\mathrm{SiO}_{2}$ nanoparticles obtained at $900 \mu \mathrm{J} /$ pulse . (b)Relationship between emission peak maximum and $\mathrm{Rh} 6 \mathrm{G}-\mathrm{SiO}_{2}$ nanoparticles concentration.

By the moving of the emission spectrum caused by the increasing of the concentration of fluorescent nanoparticles, we can certify the above explanation. Figure 4(a) shows in the GP samples normalized emission spectrum. When the pump energy is $900 \mu \mathrm{J} / \mathrm{pulse}$, the concentration of fluorescent silica nanoparticles $\left(\mathrm{Rh} 6 \mathrm{G}-\mathrm{SiO}_{2}\right)$ were $0.1,1,2,3,4$ and $5 \mathrm{wt} \%$ of. Figure (b) described the relationship between the maximum emission peak and $\mathrm{Rh} 6 \mathrm{G}-\mathrm{SiO}_{2}$ nanoparticles, from which we could see clearly that when the number of $\mathrm{Rh} 6 \mathrm{G}-\mathrm{SiO}_{2}$ nanoparticles increased. The corresponding class laser maximum peak redshifts about $18.7 \mathrm{~nm}$. In paper [14] and [15, 16], a function of Rh6G fluorescent in a gel silica substrate and colloidal solution of dye are introduced, as well as the redshift of class laser emission with the dye concentration variation. This spectrum move is defined as the existence of re-absorption ${ }^{[15,16,17]}$, or the formation of the aggregation ${ }^{[18]}$.

In order to research the possible impact of additional inert scattering body on the GP sample random laser threshold value, we study the frequency domain and time domain emission characteristics (as shown in figure 5) of GP samples, in which the silica gel contains $\mathrm{Rh} 6 \mathrm{G}-\mathrm{SiO}_{2} 4$ wt \%, $\mathrm{TiO}_{2} 0$, 0.1 and $0.8 \mathrm{wt} \%$, respectively. In order to guarantee the homogeneity of the gel sample, the size of these nanoparticles was small enough $(\sim 15 \mathrm{~nm})$.

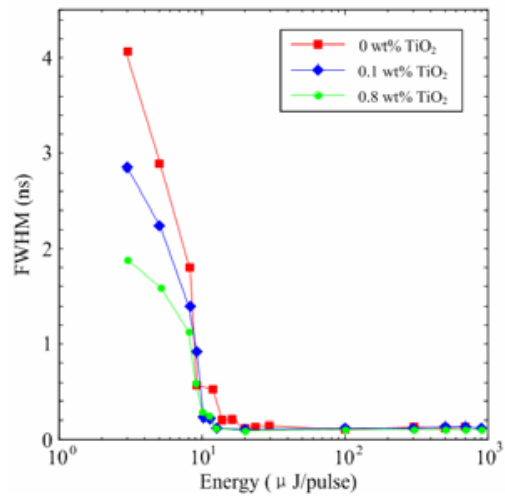

Fig. 5. In the GP samples of different silica gels containing $4 \mathrm{wt} \% \mathrm{Rh}_{6} \mathrm{G}-\mathrm{SiO}_{2}$ nanoparticles, and $0 \mathrm{wt} \%$ (squares), $0.1 \mathrm{wt} \%$ (diamonds) and $0.8 \mathrm{wt} \%$ (dots) $\mathrm{TiO}_{2}$ dispersors, the changes between FWHM of the temporal profiles and the pump pulse energy.
From the above figure, the tiny $\mathrm{TiO}_{2}$ scattering body into the corresponding GP sample has little influence on emission spectra. Whatever was the concentration of $\mathrm{TiO}_{2}$, all the line width dropped almost in the same pump energy value, i.e., the scattering effects of the inert scattered body is not able to reduce the random laser threshold. But from the relation curve between the FWHM and pump pulse energy, we can obtain that in the low pump energy value, FWHM appears significantly decrease along with the increase of the $\mathrm{TiO}_{2}$ concentration. In fact, if the $\mathrm{TiO}_{2}$ is absent in the GP samples, life time was $5 \mathrm{~ns}$; if its concentration increased to $0.1 \mathrm{wt} \%$ or $0.8 \mathrm{wt} \%$, life expectancy is 4 or $3.2 \mathrm{~ns}$. It is indicated that the sample embedded $\mathrm{TiO}_{2}$ nanoparticles in the silicon substrate occurs the non-radiative and not activated channels.

\section{CONCLUSION}

We investigate powder random medium by the theory of random laser time domain. It can be concluded that GP sample containing $4 \mathrm{wt} \% \mathrm{Rh} 6 \mathrm{G}-\mathrm{SiO}_{2}$ has a threshold of $8.5 \mu \mathrm{J} /$ pulse, which has an unconspicuous effect on random laser emission. The spectrum red-shift of GP sample at the $900 \mu \mathrm{J} /$ pulse under different concentrations of $\mathrm{Rh} 6 \mathrm{G}-\mathrm{SiO}_{2}$ gives the reason of different FWHM on low frequency pump energy. Moreover, the simulation results prove that the tiny $\mathrm{TiO}_{2}$ scattering body $(\sim 15 \mathrm{~nm})$ joined into the GP samples has a very small, even negligible effect on random laser emission. This makes an agreement with previous experiments. Above all, the random medium proposed in this paper has good stability and low threshold characteristics, which are easy to achieve and control and has a broad prospect of application.

\section{REFERENCE}

[1] V. S. Letokhov, Stimulated emission of an ensemble of scattering particles with negative absorption[J], JETP Lett. 5, 212-215 (1967)

[2] Noginov, M. A. Solid-State Random Lasers (Springer, Berlin, 2005)

[3] D. S. Wiersma, The physics and applications of random lasers[J], Nature Physics 4, 359-367 (2008)

[4] M. A. Noginov, Solid-State Random Lasers, (Springer, Berlin, 2005)

[5] [5] H. Cao, Lasing in random media[J], Waves Random Media 13, R1-R39 (2003)

[6] H. Cao, J.Y.Xu, D.Z. Zhang, Phys. Rev. Lett. 84, 5584 (2000)

[7] V.S. Letokhov, S.K. Sekatskii, Quantum Electron. 32, 1007 (2002)

[8] R.C. Polson, Z.V. Vardeny, Appl. Phys. Lett. 85, 1289 (2004)

[9] S. García-Revilla, J. Fernández, M. A. Illarramendi, et al., Ultrafast random laser emission in a dye-doped silica gel powder[J], Opt. Express $16,12251-12263(2008)$

[10] Sara García-Revilla, Marcos Zayat, Rolindes Balda, et al., Low threshold random lasing in dye-doped silica nano powders[J], Optical Society of America Opt. Express 17, 13203-13216 (2009)

[11] Jiang X. Soukoulis C M 2000 Phys. Rev. Lett. 8570

[12] Vanneste C. Sebbah P 2001 Phys. Rev Lett. 87 183903; Sebbah P, Vanneste C 2002 Phys. Rev. B 66144202

[13] Tetsu Ito, Makoto Tomita, Polarization-dependent laser action in a two-dimensional random medium[J]. Phys. Rev. B, 2002, 66(2): 027601 027605

[14] A. Anedda, C. M. Carbonaro, R. Corpino, P. C. Ricci, et al., Formation of fluorescent aggregates in Rhodamine $6 \mathrm{G}$ doped silica glasses[J], Non-Crys Sol. 353, 481-485 (2007)

[15] K. Totsuka, M. A. I. Talukder, M. Matsumoto, et al., Excitation-power-dipendent spectral shift in photoluminescence in dye molecules in strongly scattering optical media[J], Phys. Rev. B 59, 
50-53

[16] G. Beckering, S. J. Zilker, and D. Haarer, Spectral measurements of the emission from highly scattering gain media[J], Opt. Lett. 22, 1427-1429 (1997)

[17] G. Hungerford, K. Suhling, and J. A. Ferreira, Comparison of the fluorescence behaviour of rhodamine $6 \mathrm{G}$ in bulk and thin film tetraethylorhosilicate derived sol-gel matrices[J], Photochem. Photobiol. A $129,71-80$ (1999)

[18] F. Del Monte, J. D. Mackenzie, and D. Levy, Rhodamine fluorescent dimers adsorbed on the porous surface of silica gels[J], Langmuir 16, 7377-7382 (2000) 